\title{
Pour une orientation innovante et tout au long de la vie
}

Federica Minichiello

\section{OpenEdition \\ 12 Journals}

Édition électronique

URL : https://journals.openedition.org/ries/11188

DOI : $10.4000 /$ ries. 11188

ISSN : 2261-4265

Éditeur

France Education international

\section{Édition imprimée}

Date de publication : 1 décembre 2021

Pagination : 12-15

ISBN : 978-2-85420-637-9

ISSN : $1254-4590$

\section{Référence électronique}

Federica Minichiello, " Pour une orientation innovante et tout au long de la vie », Revue internationale d'éducation de Sèvres [En ligne], 88 | décembre 2021, mis en ligne le 01 décembre 2022, consulté le 26 mars 2023. URL : http://journals.openedition.org/ries/11188; DOI : https://doi.org/10.4000/ries. 11188

Ce document a été généré automatiquement le 26 mars 2023.

Tous droits réservés 


\title{
Pour une orientation innovante et tout au long de la vie
}

\author{
Federica Minichiello
}

1 Le processus d'orientation couvre tous les services proposés à des individus de tous âges, pour faire des choix éclairés et pertinents en matière d'éducation, de formation et d'emploi.

2 Le marché du travail est caractérisé par des mutations régulières, induites par des facteurs économiques, sociaux ou technologiques. On peut penser, par exemple, à l'importance accrue des réseaux professionnels ou de la mobilité professionnelle, ou aux perspectives d'innovation et de personnalisation ouvertes par l'utilisation de données massives. Ce monde en évolution implique une orientation inscrite de plus en plus dans une perspective tout au long de la vie, avec des politiques et des stratégies individuelles d'acquisition de compétences, pour plus d'adaptabilité, d'employabilité et d'épanouissement. Le Centre européen pour le développement de la formation professionnelle (Cedefop) estime qu'environ 128 millions d'adultes, dans l'Union européenne, sont concernés par une situation potentielle de perfectionnement ou de reconversion professionnelle.

3 Cet article propose une sélection de publications institutionnelles récentes ${ }^{1}$ sur ce sujet, en s'intéressant en particulier à l'action des institutions européennes de référence. Les exemples de pratiques innovantes soulèvent toutefois de nombreux défis: l'anticipation des besoins en compétences, l'harmonisation des cadres de référence, la lisibilité des parcours individuels et une approche holistique des compétences, pour que l'orientation soit le plus efficace possible, et le plus adaptée aux aspirations de chacun. 


\section{Publications récentes}

\section{Lifelong guidance policy and practice in the EU: Trends, challenges and opportunities (final report)}

Ce rapport a été commandité par la Commission européenne aux universités de Warwick et de Jyväskylä. À partir de l'analyse de la littérature et de différents entretiens, les auteurs identifient onze facteurs clés dans les systèmes d'orientation tout au long de la vie. La fragmentation du secteur, au niveau décisionnel ou opérationnel, est présentée comme le principal obstacle à toute recommandation politique cohérente, car l'écosystème des acteurs d'orientation est souvent varié et complexe. Le rapport souligne deux nouvelles catégories d'acteurs clés: les professionnels de santé et de bien-être et les organismes détenteurs de données (data providers). Les systèmes d'orientation sont dispensés et financés sous plusieurs formes, avec des modèles émergents, comme dans les Flandres, où chaque citoyen peut bénéficier de crédits personnels (ou vouchers), à des intervalles de son parcours professionnel. Enfin, le déploiement du numérique dans l'orientation et, plus largement, dans l'administration (e-government) semble améliorer l'accès et la cohérence des services proposés.

https://bit.ly/3kDQ7Im

\section{Le groupe inter-agence sur l'enseignement et la formation professionnels et techniques}

5 En 2019, six organisations internationales - la Commission européenne, la Fondation européenne pour la formation (ETF), l'Organisation internationale du travail (OIT), le Cedefop, l'OCDE et l'Unesco - se sont associées pour souligner la nécessité d'investir dans l'orientation professionnelle et de démocratiser la capacité de chaque individu à «se représenter et planifier son avenir ». Leurs travaux récents indiquent que le besoin en matière d'orientation s'est accru, en raison de la pandémie de Covid-19 et des instabilités provoquées dans l'économie mondiale et sur le marché du travail. Une enquête menée auprès de responsables politiques et d'experts de 93 pays montre que l'orientation est restée un domaine peu soutenu par les mesures publiques pendant la crise, laissant les plus vulnérables et les jeunes dans des situations de grande difficulté. Parmi les exemples identifiés, le programme Primary Futures, au Royaume-Uni, destiné aux écoles primaires, facilite la mise en relation dès le plus jeune âge des élèves avec des professionnels, afin de lutter contre les stéréotypes et de mieux comprendre le lien entre études et vie professionnelle.

https://bit.ly/3kDK86B

\section{La Commission européenne}

6 La Commission s'est dotée d'un plan quinquennal afin de favoriser la montée en compétences des citoyens européens et la pertinence des actions de recrutement et de formation des entreprises. Les actions menées dans le cadre de cette stratégie comprennent une veille renforcée sur les besoins en compétences, les comptes de formation individuels, une approche européenne des micro-qualifications et la nouvelle 
plateforme Europass (https://europa.eu/europass/fr). En octobre 2021, le commissaire européen à l'emploi a lancé le déploiement des European Digital Credentials for Learning, pour une meilleure reconnaissance des qualifications et autres acquis d'apprentissage en Europe, en rappelant que «la délivrance de titres numériques constitue un changement de paradigme dans la manière dont les acquis de l'apprentissage sont fournis et partagés ». https://bit.ly/322kAJV

\section{L'OCDE}

7 L'enquête PISA 2018 montre que peu d'adolescents ont accès aux conseils dont ils auraient besoin pour leur orientation professionnelle ; leurs aspirations sont ainsi très influencées par leur environnement et leur milieu social. En moyenne, seul un étudiant sur cinq a accès aux trois activités d'orientation les plus répandues en milieu scolaire : l'échange avec un conseiller, la participation à un salon professionnel, la visite d'un lieu de travail. Le projet Career Readiness analyse les moments de transition entre école et emploi, par des études longitudinales qui suivent, dans dix pays, des cohortes d'élèves en comparant leurs expériences d'orientation, entre 14 et 16 ans, et leurs résultats sur le marché du travail, dix ans plus tard. Les résultats indiquent que les élèves ont besoin d'explorer le marché de travail. Les trois actions qui semblent avoir le plus d'impact sont les expériences de travail à temps partiel (en nombre limité d'heures), l'implication dans des associations et des temps d'observation en entreprise. Par ailleurs, les gouvernements jouent un rôle essentiel, en tant que garants de la qualification des professionnels d'orientation et facilitateurs des échanges entre le monde du travail et les écoles.

https://bit.ly/3qypkBb

\section{Le Centre européen pour le développement de la formation professionnelle (Cedefop)}

8 Les missions du Cedefop, revues en 2019, sont de «soutenir la promotion, le développement et la mise en œuvre de la politique européenne dans le domaine de l'enseignement et de la formation professionnels (EFP) ainsi qu'en matière de compétences et de qualifications, au-delà des frontières traditionnelles de l'EFP ". Parmi ses nombreuses actions, on peut citer le réseau d'experts indépendants CareersNet, qui permet de suivre l'évolution des politiques et des systèmes éducatifs dans l'espace européen, comme l'évolution vers des modèles holistiques d'orientation tout au long de la vie. En outre, Skills-OVATE est un outil d'analyse en ligne, en temps réel, des postes vacants, renseignant sur les emplois et les compétences exigées par les employeurs, qui récupère et consolide, dans des formats harmonisés, les informations publiées par des millions d'employeurs sur internet.

https://www.cedefop.europa.eu

\section{La Fondation européenne pour la formation (ETF)}

9 L'ETF aide les pays voisins de l'Union européenne et les pays d'Asie centrale à développer des systèmes d'orientation professionnelle, en adéquation avec les attentes 
du marché du travail. Un rapport récent (2020) explore les tendances en termes d'innovation dans l'orientation professionnelle : la transformation numérique est l'une des grandes tendances qui ont été identifiées, en particulier l'utilisation d'agents conversationnels (soutenue par des technologies d'apprentissage automatique) ou l'analyse de données massives pour extraire des informations en temps réel, proposer des simulateurs de carrière ou personnaliser les services d'orientation. Quelques outils sont présentés dans le rapport : une expérience pour guider les étudiants du Nigeria à travers les procédures d'admission dans les universités ou My Journey, en Irlande, qui mesure la « distance » à parcourir en termes de compétences transversales (soft skills) pour une insertion adéquate sur le marché de l'emploi, avec des objectifs et des parcours personnalisés.

https://bit.ly/30kgPP2

\section{Les défis pour bien orienter}

\section{Anticiper les compétences : le projet « Emploi des jeunes dans la région méditerranéenne » (YEM)}

Objet d'un consensus de plus en plus large, l'anticipation des besoins en compétences fournit une vision prospective des métiers et compétences de demain. Mis en œuvre par l'Unesco, le projet YEM (https://unevoc.unesco.org/yem/) a contribué à l'élaboration de modèles d'anticipation des compétences pour définir des politiques d'enseignement et de formation techniques et professionnels (EFTP). Fondé sur les résultats de la composante "emploi » des Réseaux de la jeunesse méditerranéenne (NET-MED Youth) et du projet YEM, le rapport skills forecasting in the South Mediterranean region: Approaches and lessons learned from pilot projects présente cinq modèles de prévision des compétences et leurs résultats dans sept pays de la région, en les comparant avec d'autres initiatives dans le monde. Développer de bons systèmes pour anticiper les futurs besoins de compétences nécessite du temps et des investissements considérables, en matière de production de statistiques et de données du marché, ainsi que des capacités d'analyse et de modélisation. Les États-Unis ont mis plusieurs décennies à développer de tels systèmes et peu de pays, à l'exception par exemple du Canada, montrent un lien très direct entre les projections de compétences et les actions politiques.

https://bit.ly/2YYc11q

\section{Comparer : le cadre européen des certifications (CEC)}

11 Depuis des années, de nombreux efforts ont été entrepris pour améliorer la transparence, la comparabilité et la transférabilité des qualifications entre pays et institutions. Le cadre européen des certifications (CEC) en est un exemple : créé en 2008 et révisé en 2017, il permet de « définir clairement ce qu'une personne sait, comprend et est capable de faire ", et de centraliser les informations sur les certifications en Europe, en lien avec les cadres nationaux de chaque pays. La classification européenne des aptitudes, compétences, certifications et professions (ESCO) fournit des descriptions d'environ 3000 professions et de 13000 compétences liées, traduites en différentes langues dont les langues officielles de l'Union européenne. Son objectif est 
de « soutenir la mobilité professionnelle à travers l'Europe et un marché du travail plus intégré, en offrant un langage commun sur les professions et les compétences ».

https://bit.ly/3oLfOrM ; https://ec.europa.eu/esco/portal

\section{Reconnaître : les micro-certifications}

Une approche globale des acquis d'apprentissage permet des systèmes d'orientation plus flexibles, qui tiennent compte d'une richesse d'apprentissages et d'expériences, dans tous contextes : formel, non formel et informel. L'évolution du marché du travail laisse d'ailleurs présager l'émergence de certifications de plus en plus courtes, voire de micro-certifications. L'article "Les micro-certifications : enjeux, défis et initiatives ${ }^{2}$ " présente les enjeux sous-jacents aux micro-certifications et les mesures pour les mettre en œuvre, avec des exemples d'initiatives à travers le monde: Australie, Canada, Nouvelle-Zélande, etc.

https://bit.ly/3orNIBK

\section{Échanger : un espace de données au sein de GAIA-X}

13 Pour des services d'orientation réellement innovants et personnalisés, il est nécessaire de disposer de données suffisantes et de qualité. Malgré les efforts d'harmonisation et de passerelle entre référentiels et cadres nationaux, les données liées aux compétences restent dispersées entre de nombreux acteurs, dans différents systèmes d'information et de classification. Cette fragmentation réduit la possibilité de recommandation et d'accompagnement sur mesure qui permettrait une orientation efficace. L'initiative européenne GAIA-X vise à promouvoir un écosystème éthique, de confiance et sécurisé, qui permet la circulation et l'utilisation des données. Un espace de données spécifique pour l'éducation et les compétences tout au long de la vie est en cours de constitution, afin de faire émerger de nouveaux services éducatifs à l'aide de dispositifs adaptatifs et intelligents. L'orientation est l'une des priorités de ce futur data space.

https://bit.ly/324F2K8

\section{NOTES}

1. Sitographie arrêtée le $1^{\text {er }}$ novembre 2021.

2. H. Beaucher (2021). «Les micro-certifications : enjeux, défis et initiatives » [en ligne]. Article publié sur le portail documentaire LISÉO de France Éducation International. 
INDEX

Mots-clés : orientation professionnelle, formation tout au long de la vie, innovation, marché du travail, cadre national de certification

Palabras claves : orientación profesional, formación permanente, innovación, mercado de trabajo, marco nacional de cualificaciones

Keywords : vocational guidance, lifelong training, innovation, labour market, national qualifications framework

\section{AUTEUR}

\section{FEDERICA MINICHIELLO}

Federica Minichiello est directrice du Laboratoire d'innovation et de ressources en éducation (LIRE) de France Éducation International. Courriel : minichiello[at]france-education-

international.fr 